\title{
Histonedeacetylase 1 mRNA has elevated expression in clinical specimen of bladder cancer
}

\author{
Alivand $\mathrm{M}^{1}$, Soufi $\mathrm{RT}^{1}$, Madani $\mathrm{AH}^{2}$, Esmaeili $\mathrm{SN}^{2}$, Vaziri $\mathrm{HR}^{3}$, Sohani $\mathrm{MM}^{4}$, Rafati $\mathrm{M}^{5}$, \\ Hamami $\mathrm{P}^{6}$, Ajamian $\mathrm{F}^{3}$
}

Department of Biology, Faculty of Sciences, University of Guilan, Rasht, Iran. ajamian@guilan.ac.ir

\begin{abstract}
OBJECTIVE: HDACs are among transcriptional regulatory elements that regulate key features of proliferation and differentiation in all cell types including cancerous. They may also interfere in such stages of cancer development as migration, invasion, multi-drug resistance and angiogenesis. Proven information about HDAC1 role in development of bladder cancer is limited only to cell lines in vitro. The lack of a comprehensive clinical in vivo study led us to evaluate HDAC1 expression in human clinical specimens.

METHODS: We analyzed a large group of bladder cancer patients. The presence of hHDAC1 mRNAs were tracked using specific HDAC1 primers in cancer samples and the quantity of HDAC1 transcripts were quantified using real time qPCR method and was compared to those of normal bladder samples from healthy patients. RESULTS: HDAC1 mRNA expression was significantly elevated in Bladder cancer specimens. To our knowledge, this result is the first, showing an elevation in vivo in HDAC1 mRNA levels in clinically cancerous tissue of patients with bladder cancer.

CONCLUSIONS: We conclude that hHDAC1 overexpression might be implicated in bladder cancer tumorigenesis and that the over-expressed HDAC1 mRNA might be a potential diagnostic marker and, a target for treatment of bladder cancer using HDACi-drugs in future (Tab. 2, Fig. 2, Ref. 30). Text in PDF www.elis.sk.

KEY WORDS: histone deacetylase1 (HDAC1) mRNA, histone deacetylase inhibitors (HDACi), bladder cancer.
\end{abstract}

\section{Introduction}

Bladder cancer is the 9 th most frequent cancer in men and the 17th most frequent cancer in women (1). Populations of North America, Northern Africa and Europe have the highest incidence rates of this malignancy. Most of bladder cancer cases are superficial subtype, while approximately $20-40 \%$ of the patients will develop invasive cancer. Bladder cancer includes heterogeneous morphological categories: More than $90 \%$ of bladder cancer cases are urothelial carcinoma, whereas adenocarcinoma, squamous cell carcinoma, small cell carcinoma and other subtypes are rare (2). Although around $20 \%$ of non-invasive and stroma invasive tumors are cured, $60-70 \%$ recur at least once in 5 years and 10-20\% progress to muscle invasive cancer after the first removal (3). Epi-

${ }^{1}$ International Pardis Unit, University of Guilan, Rasht, Iran, ${ }^{2}$ Urology Research Center, Razi Hospital \& Guilan University of Medical Sciences, Rasht, Iran, ${ }^{3}$ Department of Biology, Faculty of Sciences, University of Guilan, Rasht, Iran, ${ }^{4}$ Department of Cultivation, Faculty of Agriculture, University of Guilan, Rasht, Iran, ${ }^{5}$ Department of Family Medicine, Faculty of Medicine and Dentistry, University of Alberta, Edmonton, Canada, and ${ }^{6}$ Eye Research Center, Iran University of Medical Sciences, Tehran, Iran

Address for correspondence: F. Ajamian, PhD, Department of Biology, Faculty of Science, University of Guilan, C.P., 41335-1914, Namjoo St., Rasht, Iran.

Phone/Fax: +98.13.33333647

Acknowledgment: This study was sponsored by University of Guilan. genetic events such, as histone acetylation/deacetylation, control transcriptional status of genes that play vital roles in development of the cancer (4). Acetylation and deacetylation occur by function of Histone Acetyl Transferases (HATs) and Histone Deacetylases (HDACs), respectively. Whereas acetylation induces remodeling of core nucleosome and transcriptional activation, the deacetylation of histone tails results in chromatin condensation, which in turn, causes repression of transcription (5). N-terminal tails of the core histones $\mathrm{H} 2 \mathrm{~A}, \mathrm{H} 2 \mathrm{~B}, \mathrm{H} 3$ and $\mathrm{H} 4$ are the best known substrates for these enzymes. Non-histone proteins can also be altered in their function by acetylation and deacetylation (6). HDACs are grouped into four classes of I, II, III and IV (7). Eleven family members of Class I, II and class IV are called as "classical" HDACs, whereas class III members are named Sirtuin proteins (7). Inside HDAC family, the cellular and biochemical functions of HDAC1 are relatively well studied. HDAC1 is a highly conserved enzyme presented in the cell nucleus of all tissues and, participates in both proliferation and cell death (8). This enzyme is associated with nuclear multi protein complexes that are important elements for repression of transcription and epigenetic modifications (9). This means that $\mathrm{HDAC} 1$ acts in a complex enzymatic structure. Besides HDAC1, it has recently been shown that other HDACs might also interfere in such cancerous cells functions such as: differentiation, cell cycle arrest and apoptosis, as well as migration, invasion, multi-drug resistance and angiogenesis. Apparently, functions of HDACs are not redundant and the cancer cells clearly are unable 
to compensate an absence of a given HDAC (10). According to the most studies, HDAC1 has a crucial role in developing and progression of different human cancer types and its over-expression may be associated with tumor growth and a poor prognostic factor in many malignancies (11). Over-expression of HDAC1 has been reported in gastric, pancreatic and prostatic cancers and, it is shown to be a prognostic factor in breast and prostatic tumors (4, 11-13). In exocrine pancreatic epithelial cancer, cells lacking HDAC1 are enlarged and flattened with an irregular shape with additionally cytoplasmic vacuoles and micronuclei (4). It seems HDAC1 is needed for inhibiting the cancer cells from starting a state of permanent cell division stop, such as: mitotic catastrophe and cellular senescence (14). Acetylation of histones $\mathrm{H} 3$ and $\mathrm{H} 4$ and regulating expression of CDK inhibitors are among mechanisms of HDAC1, which mediates these functions (4). Many researchers introduced class I HDACs as the most functional target for cancer therapy because HDAC inhibitors (HDACi) with the activity against class I family usually present major anti-proliferative and apoptosis-inducing effects (10). In addition, anti-tumor effects of HDACi might be extended by blocking deacetylation of various proteins involved in tumorigenesis (15). Such effects caused by HDAC inhibitors are of great interest of developing HDAC inhibitors as novel anticancer drugs and several HDACi have recently been introduced, which selectively inhibit only the class I enzymes; especially HDAC1 (16). Various other HDAC inhibitors are currently being tested in clinical trials and some have been approved for clinical treatments by FDA (U.S. Food and Drug Administration) (17).

Our goal in this study was to evaluate HDAC1 mRNA expression changes in Bladder tissues in patients with cancer. We have analyzed a large clinically characterized group of patients with bladder cancer and measured the expression levels of HDAC1 mRNA in clinical specimen. Based on our result, we speculate that in the future, HDAC1 can be introduced as a prognostic biomarker in the bladder cancer diagnosis and, a potential HDACi-drug target in the treatment for this type of cancer.

\section{Material and methods}

\section{Patient characteristics}

88 patients diagnosed with bladder cancer at Razi and Aria hospitals in Rasht, Guilan, Iran, aged from 42 to 90 years old (median 65.5) participated in the current study. Of that, 76 were male and 12 were female. 79 samples were collected from normal bladder tissues of 66 men and 13 women, aged from 38 to 87 years (median 61.3 years old). All patients signed an informed consent in order to give the permission of using their Bladder tissues. This study has been approved by Guilan Medical Sciences University Ethics Committee.

\section{Tissue and clinicopathological data}

Cancerous tissues of bladder were collected after Transurethral Resection (TUR) and/or biopsies and normal tissues of bladder were collected after "scrutiny" biopsies. Fresh tissues were snap-frozen in liquid nitrogen and stored at $-80{ }^{\circ} \mathrm{C}$ until entering
Tab. 1. Clinicopathologicalcharacteristics of bladder cancer patients.

\begin{tabular}{lc}
\hline Characteristic & No of cases (\%) \\
\hline Sample number & 88 \\
\hline Mean \pm Standard deviation & 65.5 \\
\hline Age (Year) & \\
$\quad$ Range & $42-90$ \\
\hline Gender, n (\%) & $12(13)$ \\
$\quad$ Female & $76(87)$ \\
$\quad$ Male & \\
Grading, n (\%) & $51(58)$ \\
$\quad$ High grade Tumor & $37(42)$ \\
$\quad$ Low Grade Tumor & \\
\hline Muscle invasion, $\mathrm{n}(\%)$ & $18(20)$ \\
$\quad$ Present & $70(80)$ \\
$\quad$ Absent &
\end{tabular}

laboratory process. Systematic histopathological analysis of the biopsies was performed in the same time as the freezing in liquid nitrogen. All bladder tumor samples were examined to determine the grade of respective Bladder carcinoma. Of 88 cancer samples, 37 showed low grade tumors whereas 51 were diagnosed to be higher grade. Clinicopathological data of the patients in current study showed that muscle invasion occurred in 18 patients, while it was absent in the rest of 70 cases. Control samples were from "scrutiny" biopsies of normal bladder parenchyma collected from the patients with bladder objection, but without showing any type of bladder cancers. A part of normal bladder control samples were obtained through open transurethral prostatectomy. The cancerous samples were categorized into a low-to high grade groups according to the 2004 WHO (World Health Organization) classification of urothelial neoplasia. Clinicopathological data of the patients of this study is given in the Table 1 .

\section{Quantitative real-time PCR}

The fresh specimens from patients were collected to determine HDAC1 expression alterations in cancerous bladder tissues versus samples from healthy tissues. Only pathologically confirmed tissues were considered cancerous and were taken to analysis.

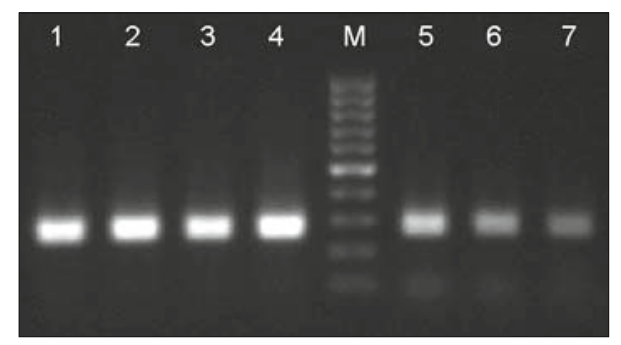

Fig. 1. Agarose gel electrophoresis of PCR products stained by Ethidium Bromide: Lines 1 to 4 show hHDAC1 fragments, amplified from randomly picked cancerous bladder samples; Lines 5 to 7 show different $h H D A C 1$ products, amplified from randomly picked normal bladder tissues. The original total RNAs amounts of all shown samples and their corresponding loaded materials are considered to be equal. The length of all PCR product is $263_{b p}$. Lane M shows $100_{b p}$ DNA marker (Fermentase, Lithuania). 
Tab. 2. Primer names, corresponding sequences and product sizes.

\begin{tabular}{llc}
\hline Primer & Sequence & Product size $(b p)$ \\
\hline HDAC1 & F: 5'- TGGCCATCCTGGAAGACGA-3' & \multirow{2}{*}{263} \\
& R: 5'-CATGACCGGCTTGAACCACC-3' & \\
\hline GAPDH & F: 5'-CAACTGCTTAGCACCCCTGG-3' & \multirow{2}{*}{280} \\
& R: 5'-GCAGGTCAGGTCCACCACTG-3' & \\
\hline 18S rRNA & F: 5' CGGCTACCACATCCAAGGAA-3' & \multirow{2}{*}{187} \\
& R: 5'- GCTGGAATTACCGCGGCT-3' & \\
\hline
\end{tabular}

$\mathrm{F}$ - forward and, $\mathrm{R}$ - reverse primers

Total RNAs were extracted from previously frozen Bladder tissues by TRIzol reagent (Invitrogen, USA) according to the manufacturer's instruction. The quality and quantity of extracted RNAs were specified by spectrophotometer (Kruss, Germany) at A260 and A280 nm wavelengths. The RNA sample integrities were confirmed using measurement of relative sharpness of $28 \mathrm{~S}$ to $18 \mathrm{~S}$ ribosomal RNA bands in agarose gel electrophoresis. One to two micrograms of total RNA from each sample were hybridized to Oligo(dT) primers and reverse-transcribed to cDNA by reverse transcriptase enzyme using cDNA synthesis kit (Thermo Scientific, USA). Suitable and equal amounts of each single stranded cDNAs were prepared and then subjected to quantitative real-time Polymerase Chain Reactions in triplicate dilutions using SYBR Green PCR Master Mix in CFX96 ${ }^{\mathrm{TM}}$ Real-Time PCR Detection Systems (Bio-Rad, USA). At least two human housekeeping genes of GAPDH and 18S rRNA were used to calculate and normalize relative gene expression data.

Before performing a real-time quantitative PCR, the standard PCR products amplified using hHDAC1 primers were surveyed by either agarose gel electrophoresis against DNA sized markers or by sequencing, to determine the specificity of PCR products amplified (Fig. 1). The samples were also PCR amplified with at least two set of GAPDH and 18S rRNA primers.

All primers were designed using either NCBI primer design tool (National Center $f$ or Biotechnology Information, USA) or Primer3-BioTools (University of Massachusetts, USA). The nucleic acid sequences of the primers used in this study are mentioned in the Table 2. The thermo-cycling conditions of real-time qPCR for hHDAC 1 primers were $95{ }^{\circ} \mathrm{C}$ for 10 min followed by 35 to 40 cycles of $95{ }^{\circ} \mathrm{C}$ for $30 \mathrm{~s}$ and $60{ }^{\circ} \mathrm{C}$ for $45 \mathrm{~s}$.

For q-PCR, each sample was run in triplicate and each experiment was performed two times. The mean threshold cycle $(\mathrm{Ct})$ value for each assay was used for next calculations. This value is the PCR cycle number, at which the fluorescence emission transits the threshold intersects with the exponential region of the amplification curve by the CFX Manager Software (Bio-Rad). Comparative $\mathrm{Ct}$ value method was the choice for the analysis of the results. The $\mathrm{Ct}$ values were normalized to the GAPDH and $18 \mathrm{~S}$ rRNA expression values by subtracting $\mathrm{Ct}$ means of used housekeeping genes, from $\mathrm{Ct}$ means of target gene for each sample to get the $\Delta \mathrm{Ct}$ mean. The $\Delta \mathrm{Ct}$ mean values were then changed into fold change values, based on a hypothesis of doubling of PCR product every cycle, according to the manufacturer's guidelines.
Statistical analysis

Quantitative data are presented as the mean \pm SD (Standard Deviation). Statistical analyses were performed with the use of SPSS 16.0 software. The significant differences between the control and test groups were analyzed by Student's t-test. p value < 0.05 was considered as statistically significant.

\section{Results}

HDAC1 mRNA expression levels were quantified in fresh human bladder cancer specimen using the relative quantification method of real-time quantitative PCR. Brief clinicopathological characteristics of cancer patients are summarized in the Material and Methods part. In this investigation, only pathologically confirmed tissues were considered cancerous and were taken to analysis of HDAC1 mRNA expression. We found -after normalizing our target hHDAC1 mRNA expression quantities against those of corresponding housekeeping genes- that the mRNA levels of hHDAC1 gene in malignant bladder tissues was clearly and significantly higher than in those of normal healthy control samples i.e. $5.6(0.41)$ vs. 1 (0.41) of relative expression (Fig. 2) $(\mathrm{p}<0.05$, Student's $t$-test). This means that hHDAC1 mRNA expression of cancerous tissues was raised more than 5 folds in comparison to that of normal healthy tissues. This result was obtained from randomly picked cancerous samples, regardless of samples' characterization such as: their grads, genders or ages. The control samples were the healthy normal bladder parenchyma biopsies as described in the Material and methods. In addition to healthy biopsy samples, parts of normal bladder control samples were obtained through open transurethral prostatectomy. We were aware that these urothelial samples might not truly be free from inflammatory process associated with prostatic hyperplasia and to address this, we did not use the corresponding tissues in the vicinity of bladder carcinomas as the main normal control samples for current calculation. However, as we were curiously speculating, the results using such corresponding tissues were expectedly similar to those values

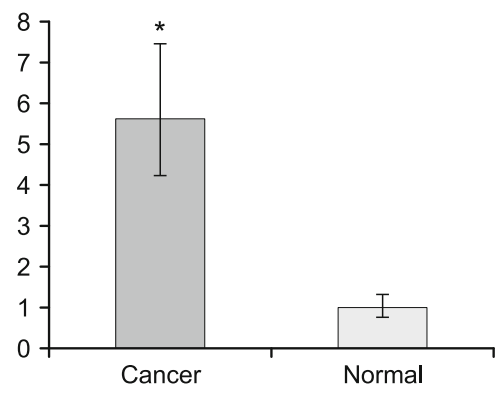

Fig. 2. hHDAC1 mRNA relative expression in cancer specimen in comparison to normal human bladder tissues. The result shown in graph is normalized to at least two housekeeping genes expressions amounts. The control samples shown in this graph are normal biopsy samples. Each column shows the mean relative value of mRNA levels from all triplicate samples for Real Time Quantitative PCR. Bars represent the mean \pm SD. A significant difference in hHDAC1 mRNA expression level is shown between cancerous and normal tissues according to Student's t-test $(* \mathbf{p}<\mathbf{0 . 0 5})$. 
calculated using biopsy normal control samples (data not shown). No correlation with the patient genders, tumor muscle invasion, age or grading of the tumors were found when each of these different samples were studied separately comparing their relative expression levels to those of control samples (data not shown).

\section{Discussion}

Histone acetylation is a reversible process regulated by histone acetyl transferases (HATs) and histone deacetylases (HDACs). It is an epigenetic mechanism, which is involved in chromatin remodeling and translational regulation. Improper function of HDACs, including their overexpression, plays a vital role in developing of many type of cancers.

As HDAC inhibitors (HDACi) have been introduced as promising new agents for cancer therapy, there is a growing interest in studying of which type, class or member of HDACs might be involved in emergence or development of various human cancer types. In particular, the class I of HDACs has been the center of attention (11). Previous proven reports indicating HDAC1 involvement in progression of different types of human solid cancerous tumors motivated us to study HDAC1 as candidate isoform of class I of HDACs enzymes in bladder carcinoma formation $(4,11)$. HDACs over-expression and the inhibitory effects of HDAC inhibitors on bladder cancer progression have been shown only in cell lines in vitro (18), however, no study has been conducted in HDAC mRNAs levels, including HDAC1 changes in vivo in clinical bladder cancer specimens. Expression of histone deacetylase 1, 2 and 3 proteins has recently been shown in clinical urothelial bladder cancer cases (19). However, to our knowledge; our work is the first one to measure expression alteration of hHDAC1 transcripts in human bladder cancer tissues in vivo. Upregulation of hHDAC1 mRNA in vivo in our results indicates that HDAC1 may play an important role in human bladder cancer emergence/development. This conclusion has the support of other recent studies showing obvious correlations between overexpression of selected HDACs and several clinicopathological characteristics of some human malignancy such as breast cancer and, their tumor growth size and patient survival rates (11). Over expressed HDAC1 protein in colon, lung and gastric cancers cells contributes to an aggressive behavior and poor prognosis (20-22). Likewise, alteration in HDAC1 expression levels were found in other human tumors including endometrial, ovarian, pancreatic cancers, as well as in hepatocellular carcinoma tumors, where the elevated expression of HDAC1 is usually associated with a poor prognosis (23-25). Strong HDAC1 overexpression in prostate cancer has been accompanied by cancer progression and poor prognosis (12). In addition, the group of studies have analyzed the effect of different HDAC inhibitors in treatment of Bladder cancer cell lines: for example, HDAC inhibitors of MS-275 and TSA have been shown to be able to block the cell cycle in T24 cell line and induce apoptosis (18). Another study in 2012 showed that Valproic acid; a HDAC inhibitor decreased urothelial cancer cell proliferation (27). These studies suggest that HDACs expression may play an important role in bladder cancer occurrence. The recent study showed an improper increase in the expression and recruitment of
HDAC1 results in aberrant regulation of specific genes involved in cell cycle and cell survival, leading to development of invasive adenocarcinoma of pancreas (4). Therefore, it has been suggested that HDAC1 is a potential prognostic biomarker and therapeutic target in pancreatic adenocarcinoma (4). Another study showed a decrease in colon cancer cells proliferation as the result of HDAC1 gene knockdown (28). Additionally, a higher HDAC expression level has been associated with a poor prognosis in Gastric cancer (29).

Recently, the study of HDAC1 expression in testicular germ cell tumors revealed a significant difference between seminomaand non-seminomatous germ cell tumors, while no correlation with prognosis was shown (30). In 2011, Patani et al demonstrated that HDAC1 was overexpressed in breast cancer compared to normal or benign breast tissues. Moreover, higher HDAC1 expression was accompanied with higher grades of breast tumor and thus this study suggested that HDAC1 over expression is an important prognostic factor in breast cancer development (11). Up to now, most studies of HDAC1 expression in malignant tumors suggest a probable correlation between overexpression of this enzyme and tumor growth, progression and being a poor prognostic factor in these malignancies. Our finding in studying the human bladder cancer is in agreement with other studies of these types.

Collectively, our results showed HDAC1 mRNA level was elevated in bladder tissues of patients with bladder cancer. In accordance with the similar results of other researchers, the findings in the bladder cancer cell line and in other types of cancer cells and tissues might indicate the importance of tight and proper regulation of HDAC1 gene expression for normal function of bladder cells in healthy people and the fact that an overexpression of this gene may trigger bladder cancer tumorigenesis in human.

An important strategy in developing of future cancer treatment involving HDAC inhibitors is the identification of the most relevant HDACs causing tumorigenesis in a given tumor, in an individual patient and, subsequently the selection of proper combination of compounds from a library of selective HDAC inhibitors. Through this finding we may suggest that HDAC1 is a potential molecule to be targeted by a given anticancer HDACi drug and this study is the first step in the pathway of constructing such selective scheme for HDACi bladder cancer therapy. The specific histone deacetylase inhibitors albeit remain to be discovered and further studies together with the results of this study may become useful tools for clinical applications such as: diagnosis, treatment and prognosis of bladder cancer, as well as designing and developing of new antibladder cancer drugs. Since the HDACs family genes also include several other members, it would be important to clarify the association between the expression level of the individual HDACs and the bladder cancer occurrence for each HDAC(s), in order to provide a better insight to the roles, in which each specific HDAC(s) may play in the development of bladder cancer. The work described in this study was focused on determining of just HDAC1 expression in bladder cancer. Likewise, this study only focused on HDAC1 gene expression on mRNA level and thus, these results are not accompanied by immunochemistry data.

Although for heterogeneous urothelial carcinoma it might be considered unusual, we were not able to find a significant differ- 
ence between HDAC1 expression in different patient genders and ages when each of these different samples was studied separately comparing to their relative expression levels to those of the control samples (data not shown). These results reflect a quick hHDAC1 possible involvement in developing human bladder cancer tumorigenesis and thus, we did not follow-up patients' clinical data in long term to see whether there is any correlation between the mRNA expression levels with survival rates and/or prognosis. Despite, the result of this study is the first reported of HDAC1 mRNA over-expression in clinical tissues of bladder cancer in vivo and suggests that overexpression of the human HDAC1 may be correlated with the incidence of bladder cancer in human.

\section{Learning points}

As an "epigenetic" molecule, HDAC1 of class I of HDACs has a proven transcriptional regulatory role in cell-cycle arrest/ proliferation in both cancerous bladder cell lines in vitro and in vivo in human clinical specimens, at mRNAs level.

This finding is interesting because it shows that HDAC1 could play as a potential diagnostic marker and can be considered as the target molecule for the treatment with anti-cancer drugs of Histonedeacetylas inhibitors (HDACi) in further bladder cancer treatment.

\section{References}

1. World Cancer Report 2014. World Health Organization. 2014; pp. Chapter 1.1.

2. Lopez-Beltran A, Cheng L. Histologic variants of urothelial carcinoma: differential diagnosis and clinical implications. Hum Pathol 2006; 37 (11): 1371-1388

3. Herr H, Lamm DL, Denis L. Management of Superficial Bladder Cancer, Chapter 26. Princip Pract Genitourin Oncol 1997; 273.

4. Zhou W, Liang IC, Yee NS. Histone deacetylase 1 is required for exocrine pancreatic epithelial proliferation in development and cancer. Cancer Biol Ther 2011; 11 (7): 659-670.

5. Monneret C. Histone deacetylase inhibitors. Eur J Med Chem 2005; 40 (1): $1-13$.

6. Kristensen LS, Nielsen HM, Hansen LL. Epigenetics and cancer treatment. Eur J Pharmacol 2009; 625 (1-3): 131-142.

7. Gregoretti IV, Lee YM, Goodson HV. Molecular evolution of the histone deacetylase family: functional implications of phylogenetic analysis. J Mol Biol 2004; 338 (1): 17-31.

8. Yang XJ, Seto E. The Rpd3/Hda1 family of lysine deacetylases: From bacteria and yeast to mice and men. Nature Rev Mol Cell Biol 2008; 9 (3): 206-218.

9. Huang Y, Myers SJ, Dingledine R. Transcriptional repression by REST: recruitment of $\operatorname{Sin} 3 \mathrm{~A}$ and histone deacetylase to neuronal genes. Nature Neurosci 1999; 2 (10): 867-872.

10. Olaf Witt, Hedwig E. Deubzer, Till Milde, Ina Oehme. HDAC family: What are the cancer relevant targets? Cancer Lett 2009; 277 (1): 8-21.

11. Eom M, Oh SS, Lkhagvadorj S, Han A, Park KH. HDAC1 Expression in invasive ductal carcinoma of the breast and its value as a good prognostic factor. Korean J Pathol 2012; 46 (4): 311-317.

12. Song Y, Shiota M, Tamiya S, Kuroiwa K, Naito S, Tsuneyoshi M. The significance of strong histone deacetylase 1 expression in the progression of prostate cancer. Histopathology 2011; 58 (5): 773-780.
13. Mutze K, Langer R, Becker K et al. Histone Deacetylase (HDAC) 1 and 2 Expression and Chemotherapy in Gastric Cancer. Ann Surg Oncol 2010; 17 (12): 3336-3343.

14. Okada H, Mak TW. Pathways of apoptotic and non-apoptotic death in tumor cells. Nature Rev Cancer 2004; 4 (8): 592-603.

15. Weichert W, Roske A, Gekeler V et al. Histone deacetylases 1, 2 and 3 are highly expressed in prostate cancer and HDAC2 expression is associated with shorter PSA relapse time after radical prostatectomy. Brit J Cancer 2008; 98 (3): 604-610.

16. Bolden JE, Peart MJ, Johnstone RW. Anticancer activities of histone deacetylase inhibitors. Nature Rev Drug Discov 2006; 5 (9): 769-784.

17. Prince HM, Bishton MJ, Harrison SJ. Clinical studies of histone deacetylase inhibitors. Clin Cancer Res 2009; 15 (12): 3958-3969.

18. Qu W, Kang YD, Zhou MS, Fu LL, Hua ZH, Wang LM. Experimental study on inhibitory effects of histone deacetylase inhibitor MS-275 and TSA on bladder cancer cells. Urol Oncol 2010; 28 (6): 648-654.

19. Poyet $C$, Jentsch B, Hermanns $T$ et al. Expression of histone deacetylases 1, 2 and 3 in urothelial bladder cancer. BMC Clin Pathol 2014; 14 (1): 10. doi: 10.1186/1472-6890-14-10.

20. Higashijima J, Kurita N, Miyatani T et al. Expression of histone deacetylase 1 and metastasis-associated protein 1 as prognostic factors in colon cancer. Oncol Rep 2011; 26 (2): 343-348.

21. Sudo T, Mimori K, Nishida N et al. Histone deacetylase 1 expression in gastric cancer. Oncol Rep 2011; 26 (4): 777-782.

22. Minamiya Y, Ono T, Saito $\mathbf{H}$ et al. Expression of histone deacetylase 1 correlates with a poor prognosis in patients with adenocarcinoma of the lung. Lung Cancer 2011; 74 (2): 300-304.

23. Weichert W, Denkert C, Noske A et al. Expression of class I histone deacetylases indicates poor prognosis in endometrioid subtypes of ovarian and endometrial carcinomas. Neoplasia 2008; 10 (9): 1021-1027.

24. Wang W, Gao J, Man XH, Li ZS, Gong YF. Significance of DNA methyltransferase-1 and histone deacetylase 1 in pancreatic cancer. Oncol Rep 2009; 21 (6): 1439-1447.

25. Rikimaru T, Taketomi A, Yamashita Y, Shirabe K, Hamatsu T, Shimada M, Maehara Y. Clinical significance of histone deacetylase 1 expression in patients with hepatocellular carcinoma. Oncology 2007; 72 (1-2): 69-74.

26. Khier H, Bartl S, Schuettengruber B, Seiser C. Molecular cloning and characterization of the mouse histone deacetylase 1 gene: integration of a retrovirus in 129SV mice. Biochim Biophys Acta 1999; 1489 (2-3): 365-373.

27. Byler TK, Leocadio D, Shapiro O, et al.,Valproic acid decreases urothelial cancer cell proliferation and induces thrombospondin-1 expression. BMC Urology 2012; 12: 21. doi: 10.1186/1471-2490-12-21.

28. Weichert W, Roske A, Niesporek S et al. Class I histonedeacetylase expression has independent prognostic impact in human colorectal cancer: Specific role of class I histone deacetylases in vitro and in vivo. Clin Cancer Res 2008; 14 (6): 1669-1677.

29. Weichert W, Röske A, Gekeler V et al. Association of patterns of class I histone deacetylase expression with patient prognosis in gastric cancer: a retrospective analysis. Lancet Oncol 2008; 9 (2): 139-148.

30. Fritzsche FR, Hasler A, Bode PK et al. Expression of histone deacetylases 1, 2 and 3 in histological subtypes of testicular germ cell tumors. Histol Histopathol 2011; 26 (12): 1555-1561.

Received August 2, 2017. Accepted August 24, 2017. 